\title{
Adverse Events of Subcutaneous Loop Recorders: Insights from the MAUDE Database
}

\author{
Joseph Heaton ${ }^{1}$, Kyrillos Rezkalla ${ }^{1}$, Jessie Fullmer ${ }^{1}$, Buniechukwuenu Mbamalu ${ }^{1}$, \\ Sivacharan Buddhavarapu ${ }^{1}$, and Samir Garyali ${ }^{1}$ \\ ${ }^{1}$ Brooklyn Hospital Center
}

June 15, 2021

\begin{abstract}
Background: Complications using internal cardiac monitors are have been reported at a low rate. Targeted analyses of complications have not been well described. Objective: To investigate and describe complications associated with internal cardiac monitor (ICM) events reported to the FDA's Manufacturer and User Facility Device Experience (MAUDE) database. Methods: Our team reviewed all reported events for the Reveal LINQ loop recorder submitted to the MAUDE database over seven years (1/1/2013-12/31/2019). A 5\% random selection of reports was audited by two researchers to ensure report validity. Two cardiologists manually reviewed death and incongruent events for final interpretation. Results: 12,652 records were obtained during the observed time period. A total of 15,587 device complications were reported. Of this, under-sensing ( $\mathrm{n}=4509$, $28.93 \%)$, premature discharge of battery $(\mathrm{n}=3262,20.93 \%)$, over-sensing $(\mathrm{n}=2788,17.89 \%)$, and other sensing issues $(\mathrm{n}=$ $1532,9.83 \%$ ) were most commonly reported. Patient adverse events were reported 1,030 times. Pain or discomfort $(\mathrm{n}=275$, $26.70 \%)$, site infection $(\mathrm{n}=213,20.68 \%)$, erosion $(\mathrm{n}=138,13.40 \%)$, and impaired healing $(\mathrm{n}=49,4.76 \%)$ were most commonly reported to affect patients. Death was reported four times; after expert review, no reports justified the device or procedure as a reasonable cause. Verification of events reported $99.53 \%$ accuracy of reporting. Conclusion: Several non-life-threatening ICM complications were commonly noted from the analysis. This study supports the safe use of ICMs. A better understanding of the complication profile will help providers select patients, provide informed consent, and expected management.
\end{abstract}

\section{Hosted file}

Manuscript Loop - JCE.docx available at https://authorea.com/users/419937/articles/526318adverse-events-of-subcutaneous-loop-recorders-insights-from-the-maude-database 